\title{
DIFFERENTIAL EQUATIONS DRIVEN BY LÉVY WHITE NOISE IN SPACES OF HILBERT SPACE VALUED STOCHASTIC DISTRIBUTIONS
}

\author{
THILO MEYER-BRANDIS
}

\begin{abstract}
We develop a white noise framework and the theory of stochastic distribution spaces for Hilbert space valued Lévy processes in order to study generalized solutions of stochastic evolution equations in these spaces driven by Lévy white noise.
\end{abstract}

\section{INTRODUCTION}

White noise analysis for Brownian motion, first introduced by Hida $[\mathrm{H}]$ and subsequently developed by him and other researchers, has been proved to be a powerful tool in different areas like f. ex. mathematical physics (see [HKPS] and references therein) or stochastic partial differential equations (see [HØUZ]). More recently it has been successfully applied in mathematical finance to generalize the Clark-Haussmann-Ocone theorem and to handle hedging situations in incomplete markets with jumps (see [AØPU], [DØP], [ØР]). Also, it serves as a useful framework for anticipative stochastic calculus. Further, in $[\mathrm{MP}]$ white noise theory is employed to study existence of strong solutions of fully non-linear SDE'S.

This increasing interest in white noise analysis has naturally been leading to different extensions of the original Gaussian setting. First extensions to a non Gaussian setting were performed in $[\mathrm{AKS}],[\mathrm{KDSU}]$. Recently, a white noise space for general Lévy processes has been constructed in [LP]. An other direction was taken from [FS] who stayed in the Gaussian framework but extended the white noise theory for $\mathbb{R}^{n}$-valued stochastic distributions as presented in [HØUZ] to $H$-valued stochastic distributions where $H$ is a general separable real Hilbert space. The authors define the white noise of an $H$-valued weak Wiener process and the corresponding white noise concepts are applied to study $H$-valued stochastic evolution equations.

The purpose of this paper is to combine $[\mathrm{LP}]$ and $[\mathrm{FS}]$ and to provide a white noise framework for Hilbert space valued Lévy processes. As an application we then look in Section 4 at stochastic evolution equations driven by additive Lévy noise with values in spaces of stochastic $H$-valued distributions of the following type

$$
\begin{aligned}
\frac{d X_{t}}{d t} & =A X_{t}+B \diamond \dot{W}_{t}, \\
X_{0} & =x \in \mathcal{D}(A) \subset S(H)_{-1}, \quad 0 \leq t<T .
\end{aligned}
$$

Here $S(H)_{-1}$ is the Kondratiev space of $H$-valued stochastic distributions, $A$ is the generator with domain $\mathcal{D}(A)$ of a $C_{0}$-semigroup $S_{t}$ on $H, B$ is a bounded operator on $H$ and $\dot{W}_{t}$ is the white noise of an $H$-valued weak Lévy process $W(t)$ (see Section 2 for definitions). For example, the stochastic Lévy noise driven heat equation

$$
\begin{aligned}
d X(t, x) & =\triangle_{x} X(t, x) d t+d W(t, x) \\
X(t, x) & =0 \text { for } t \in[0, T], x \in \partial \mathcal{O}, \quad X(0, x)=0 \text { for } x \in \mathcal{O},
\end{aligned}
$$

where $\mathcal{O}=\left\{x \in \mathbb{R}^{n} ; 0<x_{k}<a_{k}, k=1, \ldots, n\right\}$, can be represented of the form (1.1) in the Hilbert space $H=L^{2}(\mathcal{O})$ with $A=\triangle_{x}$ and $B=I$. As in the Gaussian setting one can see that only for the case $n=1$ there is an $H$-valued solution of equation (1.2). The white noise framework proposed in this paper allows for a solution in $H$-valued stochastic distribution spaces for all $n$.

Key words and phrases. White noise, Levy processes, Evolution equations, Interest rate modelling. 
Let us mention that equations of the type (1.2) could also be interpreted in the context of $\mathbb{R}$-valued stochastic distributions with $(N+1)$-parameter Lévy noise (see $[\mathrm{P}])$. One would then get for every $(t, x) \in[0, T] \times \mathcal{O} \subset \mathbb{R}^{N+1}$ an $\mathbb{R}$-valued stochastic distribution $X(t, x)$, in contrast to getting for every $t \in[0, T]$ an $L^{2}(\mathcal{O})$-valued stochastic distribution $X(t)$. In particular, when applying the Hermite transform $\mathcal{H}(X(t, x))$ (see Section 3.5 for definition) for the purpose of solving the equation one has to require strict differentiability of $\mathcal{H}(X(t, x))$ in $x$, whereas in the Hilbert space setting differentiability in $x$ is relaxed to the sense of distribution.

The remaining parts of the paper are organized as follows. In Section 2 we recall some preliminaries of Hilbert space valued Lévy processes and of white noise analysis in one dimension. In Section 3 we establish the white noise concepts around a Hilbert space valued Lévy process. We mention that we are obliged to consider Lévy processes that can be decomposed into independent components. However, from a modelling point of view, these processes are the most relevant ones. Also, we focus on pure jump Lévy processes, but the extension to processes including a Gaussian part is straight forward. The construction of the stochastic distribution spaces as well as the characterization of their elements through the Hermite transform follow closely the exposure in [FS]. Concerning the Wick product we chose a different approach than [FS] and define the Wick product between Hilbert-Schmidt operator valued distributions and $H$-valued distributions. We introduce the weak Lévy process and define the Hitsuda-Skorohod integral with respect to its white noise. We show that this integral corresponds to the Itô type integral with respect to a Hilbert space valued Lévy process in case the integrator is predictable. In Section 4 we then formulate the setting for evolution equations of type (1.1).

\section{Preliminaries}

Notation 2.1. For the whole of the paper we let $H$ be a separable real Hilbert space of dimension $N \in\{\mathbb{N}, \infty\}$. We denote by $\langle\cdot, \cdot\rangle_{H}$ the inner product and by $\|\cdot\|_{H}$ the corresponding norm in $H$ and we let $\left(h_{i}\right)_{i=1}^{N}$ be an orthonormal basis of $H$.

In this Section we quickly recall the definition and some properties of Hilbert space valued Lévy processes as well as some aspects of the white noise theory for Poisson random measures developed in $[\mathrm{LP}]$ that are going to be at the base of the next Section.

2.1. Hilbert space valued Lévy processes. Let $(\Omega, \mathcal{F}, \mathbb{P})$ be a probability space. Concerning general theory about $H$-valued random variables we refer the reader $\mathrm{f}$. ex. to the book [dPZ]. As for the special case $H=\mathbb{R}$ a Lévy process with values in $H$ is defined through the following properties:

Definition 2.2. Let $L=(L(t))_{t \geq 0}$ be a family of $H$-valued random variables defined on $(\Omega, \mathcal{F}, \mathbb{P})$. We call $L$ a Lévy process if

(1) for every $t \geq s \geq 0$ the increment $L(t)-L(s)$ is independent of the $\sigma$-algebra generated by $\{L(u): 0 \leq u \leq s\}$,

(2) for every $s, t, u \geq 0$ the increments $L(t+u)-L(t)$ and $L(s+u)-L(s)$ have the same distribution,

(3) $L(0)=0 \mathbb{P}$-a.s.,

(4) $t \longmapsto L(t)$ is continuous in probability,

(5) for $\mathbb{P}$ almost every $\omega \in \Omega$ the path $t \longmapsto L(t)(\omega)$ is càdlàg.

As we know it from Lévy processes in finite dimensional Hilbert spaces, there is also a corresponding Lévy-Khintchine formula and a Lévy-Itô decomposition into a finite variation, a Brownian and a jump part of infinite dimensional Hilbert space valued Lévy processes. For more information on this see f.ex $[\mathrm{AR}]$. For general information on finite dimensional Lévy processes we refer to [B], [Sa] or [JS]. Putting $L_{i}(t):=\left\langle L(t), h_{i}\right\rangle_{H}$ the following results can $\mathrm{f}$. ex. be found in [vG2]. 
Proposition 2.3. Let $L_{i}(t)$ be as defined above. Then

(1) for every $i=1, \ldots, N, L_{i}(t)$ is an $\mathbb{R}$-valued Lévy process,

(2) $\sum_{i=1}^{N}\left|L_{i}(t)\right|^{2}<\infty \mathbb{P}$-a.s. for all $t \geq 0$,

(3) for any $s, t, u \geq 0$ and any finite set $F \ni i$, the random vectors $\left(L_{i}(t+u)-L_{i}(t)\right.$ : $i \in F)$ and $\left(L_{i}(s+u)-L_{i}(s): i \in F\right)$ have the same distribution.

Conversely, if we have (1)-(3) above, then $L(t):=\sum_{i=1}^{N} L_{i}(t) h_{i}$ converges in $H \quad P$-a.s. for every $t \geq 0$ and $(L(t))_{t \geq 0}$ is an an $H$-valued Lévy process.

Set $\tilde{L}(t):=L(t)-E[L(t)]$, where $E[L(t)]$ denotes the expectation of $L(t)$. In the case $\tilde{L}(t)$ is a $Q$-Brownian motion where $Q$ is the covariance operator of $\tilde{L}(t)$ the orthonormal basis $\left(h_{i}\right)_{i \in N}$ consisting of eigenvectors of $Q$ decomposes $\tilde{L}(t)$ into independent $\mathbb{R}$-valued Brownian motions $L_{i}(t)$ (see [dPZ]). In the case $\tilde{L}(t)$ is a Lévy process including jumps such that $E\left[\|\tilde{L}(t)\|_{H}^{2}\right]<\infty$ for all $t \geq 0$ a corresponding decomposition into uncorrelated scalar processes can be achieved. In fact, if $Q$ is the symmetric, positive semi-definite operator of trace class uniquely defined through the relation

$$
\langle Q x, y\rangle=E\left[\langle\tilde{L}(1), x\rangle_{H}\langle\tilde{L}(1), y\rangle_{H}\right], \quad x, y \in H,
$$

then it can be shown that $t Q$ is the covariance operator of $\tilde{L}(t)$ for every $t \geq 0$. Moreover, if $\left(\lambda_{i}\right)_{i \in N}$ are the eigenvectors of $Q$ with corresponding orthonormal basis consisting of eigenvectors $\left(h_{i}\right)_{i \in N}$ and we set $\tilde{L}_{i}(t):=\left\langle\tilde{L}(t), h_{i}\right\rangle_{H}$ it follows that

$$
\text { trace } Q=E\left[\|\tilde{L}(1)\|_{H}^{2}\right]=E\left[\sum_{i=1}^{N}\left|\tilde{L}_{i}(t)\right|^{2}\right]=\sum_{i=1}^{N} \lambda_{i} .
$$

With this choice of orthonormal basis consisting of eigenvectors of $Q$ we thus get that $\tilde{L}(t)$ is decomposed in uncorrelated scalar Lévy processes $\tilde{L}_{i}(t)$, i.e.

$$
E\left[\tilde{L}_{i}(t) \tilde{L}_{j}(t)\right]=0 \quad \text { if } i \neq j
$$

2.2. White noise for Poisson random measures. In the scalar case $H=\mathbb{R}$ a white noise theory for Poisson random measures has been developed in $[\varnothing \mathrm{P}],[\mathrm{LP}]$ and $[\mathrm{L} \varnothing \mathrm{P}]$. Here, we shortly present the construction of the white noise probability space for the Poisson random measure associated to a pure jump Lévy processes from [LP] which will be the starting point in the next Section. For general information about white noise theory the reader is referred to the excellent accounts of [HKPS], [Ku] and [O].

Let $\nu(d \zeta)$ be a Lévy measure on $\mathbb{R}_{0}:=\mathbb{R}-\{0\}$, i.e. $\int_{\mathbb{R}_{0}} 1 \wedge x^{2} \nu(d \zeta)<\infty$. We denote by $\mathcal{S}\left(\mathbb{R}^{d}\right)$ the Schwartz space on $\mathbb{R}^{d}$. The space $\mathcal{S}^{\prime}\left(\mathbb{R}^{d}\right)$ is the dual of $\mathcal{S}\left(\mathbb{R}^{d}\right)$, that is the space of tempered distributions. The space $\widetilde{\mathcal{S}}(X)$ is defined as the quotient algebra

$$
\widetilde{\mathcal{S}}(X)=\mathcal{S}(X) / \mathcal{N}_{\pi},
$$

where $\mathcal{S}(X)$ is a subspace of $\mathcal{S}\left(\mathbb{R}^{2}\right)$, given by

$$
\mathcal{S}(X):=\left\{\varphi(t, \zeta) \in \mathcal{S}\left(\mathbb{R}^{2}\right): \varphi(t, 0)=\left(\frac{\partial}{\partial \zeta} \varphi\right)(t, 0)=0\right\}
$$

and where the closed ideal $\mathcal{N}_{\pi}$ in $\mathcal{S}(X)$ is defined as

$$
\mathcal{N}_{\pi}:=\left\{\phi \in \mathcal{S}(X):\|\phi\|_{L^{2}(\pi)}=0\right\}
$$

with $\pi=\nu(d \zeta) d t$. The space $\widetilde{\mathcal{S}}(X)$ is a (countably Hilbertian) nuclear algebra. We indicate by $\widetilde{\mathcal{S}}^{\prime}(X)$ its dual. 
From the Bochner-Minlos theorem we deduce that there exists a unique probability measure $\mu$ on the Borel sets of $\widetilde{\mathcal{S}}^{\prime}(X)$ such that

$$
\int_{\widetilde{\mathcal{S}}^{\prime}(X)} e^{i\langle\omega, \phi\rangle} d \mu(\omega)=\exp \left(\int_{X}\left(e^{i \phi}-1\right) d \pi\right)
$$

for all $\phi \in \widetilde{\mathcal{S}}(X)$, where $\langle\omega, \phi\rangle:=\omega(\phi)$ denotes the action of $\omega \in \widetilde{\mathcal{S}}^{\prime}(X)$ on $\phi \in \widetilde{\mathcal{S}}(X)$. We have established the white noise probability space

$$
(\Omega, \mathcal{F}, P)=\left(\widetilde{\mathcal{S}}^{\prime}(X), \mathcal{B}\left(\widetilde{\mathcal{S}}^{\prime}(X)\right), \mu\right) .
$$

By using generalized Charlier polynomials $C_{n}(\omega) \in\left(\widetilde{\mathcal{S}}(X)^{\widehat{\otimes} n}\right)^{\prime}$ (dual of the $n$-th completed symmetric tensor product of $\widetilde{\mathcal{S}}(X)$ with itself) it is possible to construct an orthogonal $L^{2}(\mu)$-basis $\left\{\mathcal{K}_{\alpha}(\omega)\right\}_{\alpha \in \mathcal{J}}$ defined by

$$
\mathcal{K}_{\alpha}(\omega)=\left\langle C_{|\alpha|}(\omega), \delta^{\widehat{\otimes} \alpha}\right\rangle,
$$

where $\mathcal{J}$ is the multiindex set of all $\alpha=\left(\alpha_{1}, \alpha_{2}, \ldots\right)$ with finitely many non-zero components $\alpha_{i} \in \mathbb{N}_{0}$. The symbol $\delta^{\widehat{\otimes} \alpha}$ denotes the symmetrization of $\delta_{1}^{\otimes \alpha_{1}} \otimes \ldots \otimes \delta_{j}^{\otimes \alpha_{j}}$, where $\left\{\delta_{j}\right\}_{j \geq 1} \subset \widetilde{\mathcal{S}}(X)$ is the following orthonormal basis of $L^{2}(\pi)$ :

$$
\delta_{k}(t, \zeta)=\delta_{k(j, l)}(t, \zeta):=\xi_{j}(t) \eta_{l}(\zeta) .
$$

Here $\left\{\xi_{j}(t)\right\}_{j}$ is the orthonormal $L^{2}(d t)$ basis consisting of Hermite functions, $\left\{\eta_{l}(t)\right\}_{l}$ is an orthonormal basis of $L^{2}(\nu)$ and $k(j, l)$ is the diagonally counting map given through

$$
k=\Delta(i, j):=\frac{(i+j)^{2}+i-j}{2} .
$$

We denote for a given $k$ the inverse image by $\Delta^{-1}(k)=:(i(k), j(k))$.

So every $X \in L^{2}(\mu)$ has the unique representation

$$
X=\sum_{\alpha \in \mathcal{J}} c_{\alpha} \mathcal{K}_{\alpha}
$$

with Fourier coefficients $c_{\alpha} \in \mathbb{R}$. Moreover we have the isometry

$$
|X|_{L^{2}(\mu)}^{2}=\sum_{\alpha \in \mathcal{J}} \alpha ! c_{\alpha}^{2}
$$

with $\alpha !:=\alpha_{1} ! \alpha_{1} ! \ldots$ for $\alpha \in \mathcal{J}$. In particular, if $\nu(d \zeta)$ is such that $\int_{\mathbb{R}_{0}} x^{2} \nu(d \zeta)<\infty$, then one can show that the family of $L^{2}(\mu)$ random variables

$$
L(t, \omega):=\left\langle C_{1}(\omega), 1_{[0, t]}(s) \zeta\right\rangle=\sum_{j \geq 1} \iint_{[0, t] \times \mathbb{R}_{0}} \delta_{j}(s, \zeta) \zeta \nu(d \zeta) d s \mathcal{K}_{\epsilon_{j}}
$$

defines a square integrable pure jump Lévy martingale with Lévy measure $\nu(d \zeta)$. Here, $\epsilon_{j}$ stands for the multiindex with all entries 0 except a 1 on the $j$ 'th place. Moreover, by the density of $\widetilde{\mathcal{S}}(X)$ and the isometry 2.9 the action of $C_{1}(\omega)$ has been extended to $f(s, \zeta)=1_{[0, t]}(s) \zeta \in L^{2}(\pi)$. The associated compensated Poisson random measure is denoted by

$$
\widetilde{N}(d t, d \zeta)=N(d t, d \zeta)-\nu(d \zeta) d t
$$

where $N(d t, d \zeta)$ is the jump measure of $L(t, \omega)$. 


\section{White Noise Framework for Hilbert Space valued Lévy Processes}

In this Section we want to introduce concepts and techniques from white noise theory that serve the purpose of studying stochastic differential equations driven by $H$-valued Lévy white noise. The case when the noise is caused by a cylindrical Brownian motion is treated in [FS] where the approach of [HØUZ] for $\mathbb{R}^{n}$ is transferred to infinite dimensional Hilbert spaces. Due to the Gaussian nature of the cylindrical Brownian motion it is in this case possible to take as in the one dimensional case the space of tempered distributions $S^{\prime}(\mathbb{R})$ as underlying white noise probability space. For a general $H$-valued Lévy process the space $S^{\prime}(\mathbb{R})$ becomes too small in order to define an appropriate white noise measure on it. One alternative would be to construct a white noise measure on the space of Hilbert space valued distribution, which is the space of all continuous functions from $S(\mathbb{R})$ to $H$. For differential equations driven by a $Q$-Brownian motion this is done in $[\mathrm{A}]$. However, we decide to take the direct product of $\tilde{S}^{\prime}(X)$ 's as introduced in Subsection 2.2 as underlying probability space because this easier allows for the introduction of a weak Lévy process, the analogue of a weak Brownian motion.

Further, we mention that we will focus on Hilbert space valued pure jump Lévy processes $\tilde{L}(t)$ where the corresponding decomposition is not only into uncorrelated but independent square integrable scalar Lévy processes $\tilde{L}_{i}(t)$. However, from a modelling point of view this is most often a reasonable assumption and thus a not too big restriction.

3.1. Abstract stochastic distributions. Let $\left(\nu_{i}(d \zeta)\right)_{i=1}^{N}$ be a sequence of Lévy measures on $\mathbb{R}_{0}$ such that for all $i$

$$
0<\lambda_{i}:=\int_{\mathbb{R}_{0}} \zeta^{2} \nu_{i}(d \zeta)<R, \quad R>0 .
$$

To each $\nu_{i}$ we associate the corresponding test function space $\tilde{S}_{i}(X)$ and white noise space $\left(\widetilde{\mathcal{S}}_{i}{ }^{\prime}(X), \mathcal{F}_{i}, \mu_{i}\right)$ as presented in 2.5. We form the direkt sum $\tilde{S}_{H}=\bigoplus_{i=1}^{N} \tilde{S}_{i}(X)$ induced with the direct product topology, and denote by $\tilde{S}_{H}^{\prime}$ its topological dual which is isomorphic to the product $\prod_{i=1}^{N} \tilde{S}_{i}^{\prime}(X)$ induced with the product topology. We put $\mathcal{F}_{H}:=\prod_{i=1}^{N} \mathcal{F}_{i}$ and $\mu_{H}:=\prod_{i=1}^{N} \mu_{i}$ and establish our underlying white noise probability space

$$
(\Omega, \mathcal{F}, P):=\left(\tilde{S}_{H}^{\prime}, \mathcal{F}_{H}, \mu_{H}\right) .
$$

The first step will be to introduce an orthogonal basis for square integrable real valued random variables $L^{2}\left(\mathbb{R}, \mu_{H}\right)$ on $(\Omega, \mathcal{F}, P)$. As in Subsection 2.6, we denote by $\left\{\delta_{i j}\right\}_{j \geq 1}$ $\subset \widetilde{\mathcal{S}}_{i}(X)$ an orthonormal basis of $L^{2}\left(d t \times \nu_{i}\right)$ for every $i$. We count the basis elements diagonally and define $\delta_{k}:=\delta_{i j}$, where

$$
k=\Delta(i, j)
$$

as in (2.8). For a multiindex $\alpha=\left(\alpha_{1}, \alpha_{2}, \ldots\right) \in \mathcal{J}$ with index $\alpha=l$ we now define $\mathcal{K}_{\alpha}(\omega) \in L^{2}\left(\mathbb{R}, \mu_{H}\right)$ in the following way: to every entry $\alpha_{k}$ determine the corresponding tuple $(i(k), j(k))$ and group the entries according to the first index $i(k)$. In this way one creates finitely many, lets say $p$, "submultiindices"

$$
\begin{aligned}
& \beta^{i_{r}}=\left(\beta_{1}, \beta_{2}, \ldots\right), \\
& i_{r}=i(k), \quad k \in \Delta\left(i_{r}, \cdot\right) \cap\{1, \ldots l\}, \quad r=1, \ldots, p,
\end{aligned}
$$

where

$$
\beta_{j}=\left\{\begin{array}{l}
\alpha_{j(k)} \text { if } k \in \Delta\left(i_{r}, \cdot\right) \cap\{1, \ldots l\} \\
0 \text { otherwise }
\end{array}\right.
$$

For $\omega=\left(\omega_{1}, \omega_{2}, \ldots\right) \in \tilde{S}_{H}^{\prime}$ we set

$$
\mathcal{K}_{\alpha}^{i_{r}}(\omega):=\left\langle C_{\mid \beta^{i_{r} \mid}}^{i_{r}}\left(\omega_{i_{r}}\right), \delta_{i_{r}}^{\widehat{\otimes} \beta^{i_{r}}}\right\rangle
$$


where $C_{n}^{i_{r}}\left(\omega_{i_{r}}\right)$ is the n-th Charlier polynomial on $\tilde{S}_{i_{r}}^{\prime}(X)$ and $\delta_{i_{r}}^{\widehat{\otimes} \beta^{i_{r}}}$ is the symmetrization of $\delta_{i_{r} 1}^{\otimes \beta_{1}} \otimes \ldots \otimes \delta_{i_{r} s}^{\otimes \beta_{s}}, s=\operatorname{index} \beta^{i_{r}}$. We then define

$$
\mathcal{K}_{\alpha}(\omega):=\prod_{r=1}^{p} \mathcal{K}_{\alpha}^{i_{r}}(\omega)
$$

By the chaos expansion result from Subsection 3.3 and the product structure of $(\Omega, \mathcal{F}, P)$ one gets like in ([HØUZ], Thm 2.2.3 \& 2.2.4) that the family $\left\{\mathcal{K}_{\alpha}(\omega)\right\}_{\alpha \in \mathcal{J}}$ constitutes an orthogonal basis of $L^{2}\left(\mathbb{R}, \mu_{H}\right)$ :

Proposition 3.1. Every $X \in L^{2}\left(\mathbb{R}, \mu_{H}\right)$ has the unique representation

$$
X=\sum_{\alpha \in \mathcal{J}} c_{\alpha} \mathcal{K}_{\alpha}
$$

with Fourier coefficients $c_{\alpha} \in \mathbb{R}$. Moreover we have the isometry

$$
|X|_{L^{2}\left(\mu_{H}\right)}^{2}=\sum_{\alpha \in \mathcal{J}} \alpha ! c_{\alpha}^{2}
$$

with $\alpha !:=\alpha_{1} ! \alpha_{1} ! \ldots$ for $\alpha \in \mathcal{J}$.

Having established a chaos expansion for real valued random variables on our probability space it is now quite straight forward to introduce a chaos expansion for $H$-valued random variables and spaces of stochastic distributions. We omit detailed proofs because they are analogous to the Gaussian case presented in [FS]. Denote by $L^{2}\left(H, \mu_{H}\right)$ the space of square integrable $H$-valued random variables. Given $F(\omega) \in L^{2}\left(H, \mu_{H}\right)$ we put $a_{i}(\omega):=$ $\left\langle F(\omega), h_{i}\right\rangle_{H}$ were $\left(h_{i}\right)_{i=1}^{N}$ was the orthonormal basis of $H$. Then $a_{i}(\omega) \in L^{2}\left(\mu_{H}\right)$ and the sum

$$
\sum_{i=1}^{N} a_{i}(\omega) h_{i}
$$

converges in $L^{2}\left(H, \mu_{H}\right)$ to $F(\omega)$. Expressing each $a_{i}(\omega)$ by its chaos expansion (3.3), say $\sum_{\alpha \in \mathcal{J}} c_{i \alpha} \mathcal{K}_{\alpha}$, yields:

Theorem 3.2. The family $\left\{\mathcal{K}_{\alpha}(\omega) h_{i}\right\}_{i=1, \alpha \in \mathcal{J}}^{N}$ is an orthogonal basis of $L^{2}\left(H, \mu_{H}\right)$. Every $F(\omega) \in L^{2}\left(H, \mu_{H}\right)$ has the unique representation

$$
F(\omega)=\sum_{i=1}^{N} a_{i}(\omega) h_{i}=\sum_{i=1}^{N} \sum_{\alpha \in \mathcal{J}} c_{i} \alpha \mathcal{K}_{\alpha}(\omega) h_{i}, c_{i} \alpha \in \mathbb{R},
$$

with corresponding isometry

$$
\|F(\omega)\|_{L^{2}\left(\mu_{H}\right)}^{2}=\sum_{i=1}^{N} \sum_{\alpha \in \mathcal{J}} \alpha ! c_{i}^{2} .
$$

This chaos expansion is now employed to define Kondratiev spaces of $H$-valued stochastic test functions and distributions. We remind that in the case of an $H=\mathbb{R}$-valued pure jump Lévy processes these spaces have been constructed in [LØP]. For $\alpha \in \mathcal{J}$ we set

$$
(2 \mathbb{N})^{\alpha}:=\prod_{i \in 1}^{\infty}(2 j)^{\alpha_{j}} .
$$

Definition 3.3. Let $\rho \in[0,1]$. Define the space of $H$-valued stochastic test functions $S(H)_{\rho}$ as all functions

$$
f(\omega)=\sum_{i=1}^{N} \sum_{\alpha \in \mathcal{J}} c_{i \alpha} \mathcal{K}_{\alpha}(\omega) h_{i}, c_{i \alpha} \in \mathbb{R}
$$


in $L^{2}\left(H, \mu_{H}\right)$ such that for all $q \in \mathbb{N}$

$$
\|f\|_{\rho, q}^{2}:=\sum_{i=1}^{N} \sum_{\alpha \in \mathcal{J}} \alpha !^{1+\rho} c_{i \alpha}^{2}(2 \mathbb{N})^{q \alpha}=\sum_{\alpha \in \mathcal{J}} \sum_{i=1}^{N} \alpha !^{1+\rho} c_{i \alpha}^{2}(2 \mathbb{N})^{q \alpha}<\infty .
$$

Definition 3.4. Let $\rho \in[0,1]$. Define the space of $H$-valued stochastic distributions $S(H)_{-} \rho$ as all formal expansions

$$
F(\omega)=\sum_{i=1}^{N} \sum_{\alpha \in \mathcal{J}} c_{i \alpha} \mathcal{K}_{\alpha}(\omega) h_{i}, c_{i \alpha} \in \mathbb{R}
$$

such that for some $q \in \mathbb{N}$

$$
\|F\|_{-\rho,-q}^{2}:=\sum_{i=1}^{N} \sum_{\alpha \in \mathcal{J}} \alpha !^{1-\rho} c_{i \alpha}^{2}(2 \mathbb{N})^{-q \alpha}=\sum_{\alpha \in \mathcal{J}} \sum_{i=1}^{N} \alpha !^{1-\rho} c_{i \alpha}^{2}(2 \mathbb{N})^{-q \alpha}<\infty .
$$

Note that we have the following representations for $f(\omega) \in S(H)_{\rho}$ respectively $F(\omega) \in$ $S(H)_{-\rho}$ :

$$
\begin{aligned}
f(\omega) & =\sum_{i=1}^{N} \sum_{\alpha \in \mathcal{J}} c_{i} \mathcal{K}_{\alpha}(\omega) h_{i}=\sum_{\alpha \in \mathcal{J}} c_{\alpha} \mathcal{K}_{\alpha}(\omega)=\sum_{i=1}^{N} f_{i}(\omega) h_{i}, \\
F(\omega) & =\sum_{i=1}^{N} \sum_{\alpha \in \mathcal{J}} c_{i \alpha} \mathcal{K}_{\alpha}(\omega) h_{i}=\sum_{\alpha \in \mathcal{J}} c_{\alpha} \mathcal{K}_{\alpha}(\omega)=\sum_{i=1}^{N} F_{i}(\omega) h_{i},
\end{aligned}
$$

where $f_{i}(\omega) \in S(\mathbb{R})_{\rho}, F_{i}(\omega) \in S(\mathbb{R})_{-\rho}$ and $c_{\alpha}=\sum_{i=1}^{N} c_{i \alpha} h_{i} \in H$. Moreover, for $q \in \mathbb{N}$

$$
\begin{aligned}
\|f\|_{\rho, q}^{2} & =\sum_{\alpha \in \mathcal{J}} \alpha !^{1+\rho}\left\|c_{\alpha}\right\|_{H}^{2}(2 \mathbb{N})^{q \alpha}=\sum_{i=1}^{N}\left|f_{i}\right|_{\rho, q}^{2}, \\
\|F\|_{\rho, q}^{2} & =\sum_{\alpha \in \mathcal{J}} \alpha !^{1-\rho}\left\|c_{\alpha}\right\|_{H}^{2}(2 \mathbb{N})^{-q \alpha}=\sum_{i=1}^{N}\left|F_{i}\right|_{-\rho,-q}^{2},
\end{aligned}
$$

where $\left|f_{i}\right|_{\rho, q}^{2}$ resp. $\left|F_{i}\right|_{-\rho,-q}^{2}$ are the corresponding norms in $S(\mathbb{R})_{\rho}$ resp. $S(\mathbb{R})_{-\rho}$.

The family of seminorms $\|f\|_{\rho, q}^{2}, q \in \mathbb{N}$ gives rise to a topology on $S(H)_{\rho}$ and it can be shown that $S(H)_{-\rho}$ is the topological dual with dual action

$$
\langle F(\omega), f(\omega)\rangle=\sum_{\alpha \in \mathcal{J}} \alpha !\left\langle b_{\alpha}, c_{\alpha}\right\rangle_{H}
$$

for $F(\omega)=\sum_{\alpha \in \mathcal{J}} b_{\alpha} \mathcal{K}_{\alpha}(\omega) \in S(H)_{-\rho}$ and $f(\omega)=\sum_{\alpha \in \mathcal{J}} c_{\alpha} \mathcal{K}_{\alpha}(\omega) \in S(H)_{\rho}$. Finally we note that we have for general $\rho \in[0,1]$

$$
S(H)_{1} \subset S(H)_{\rho} \subset S(H)_{0} \subset L^{2}\left(H, \mu_{H}\right) \subset S(H)_{-0} \subset S(H)_{-\rho} \subset S(H)_{-1} .
$$

The spaces $S(H)_{0}$ resp. $S(H)_{-0}$ are also referred to as $H$-valued Hida test function resp. Hida distribution spaces.

3.2. Some key $S(H)_{-1}$-valued processes. The purpose of the white noise theory presented in this paper is to provide a tool to solve stochastic differential equations in spaces of generalized $H$-valued stochastic processes. A generalized $H$-valued stochastic process is a function of a real variable $t \in \mathbb{R}$ that takes values in the space $S(H)_{-1}$ :

$$
F(t): \mathbb{R} \longrightarrow S(H)_{-1} \text {. }
$$

For these $S(H)_{-1}$-valued functions the usual calculus concepts of continuity, differentiability and integration in the strong sense (i.e. in the topology of $S(H)_{-1}$ ) apply. For a more detailed description of how these concepts are expressed through the corresponding 
chaos expansion we refer to [FS] where the in this regard completely analogous Gaussian case is treated. We now want to introduce some key generalized processes in the context of white noise theory for Lévy processes: to our family $\left(\nu_{i}(d \zeta)\right)_{i=1}^{N}$ of Lévy measures we construct the corresponding the $H$-valued Lévy process (if existing), the weak Lévy process and its singular white noise process.

Example 3.5. (H-valued Lévy process) As becomes clear from Proposition 2.3, a square integrable pure jump $H$-valued Lévy process is characterized through the family of Lévy measures $\left(\nu_{i}\right)_{i=1}^{N}$ corresponding to its decomposition into scalar Lévy processes such that

$$
\sum_{i=1}^{N} \int_{\mathbb{R}_{0}} \zeta^{2} \nu_{i}(d \zeta)<\infty .
$$

Lets assume condition (3.5) is valid for our family $\left(\nu_{i}\right)_{i=1}^{N}$. We put

$$
L_{i}(t)=L_{i}(t, \omega):=\left\langle C_{1}^{i}(\omega), f^{i}\right\rangle_{H}, \quad i=1, \ldots, N,
$$

where $f^{i}(s, \zeta)=\left(0, \ldots, 0,1_{[0, t]}(s) \zeta, 0, \ldots\right)$ with $1_{[0, t]}(s) \zeta$ on the $i$-th place. Then $\left\{L_{i}(t)\right\}_{i=1}^{N}$ is a family of independent $R$-valued pure jump, square integrable Lévy martingales. Each $L_{i}(t)$ has Lévy measure $\nu_{i}$ with corresponding isometry

$$
E\left[L_{i}(t)^{2}\right]=t \int_{\mathbb{R}_{0}} \zeta^{2} \nu_{i}(d \zeta)<t R
$$

Then by Proposition 2.3

$$
L(t)=L(t, \omega):=\sum_{i=1}^{N} L_{i}(t) h_{i}
$$

is a square integrable $H$-valued Lévy process with covariance operator $Q$ that has eigenvalues $\lambda_{i}=\int_{\mathbb{R}_{0}} \zeta^{2} \nu_{i}(d \zeta)$ and eigenvectors $h_{i}$. We can derive its chaos expansion by using expansion 2.11 on every $L_{i}(t)$ :

$$
\begin{aligned}
L(t) & =\sum_{i=1}^{N} \sum_{j=1}^{\infty}\left(\iint_{[0, t] \times \mathbb{R}_{0}}\left(\delta_{j i} \zeta\right) \nu_{i}(d \zeta) d s\right) \mathcal{K}_{\epsilon_{\Delta(i, j)}} h_{i} \\
& =\sum_{i=1}^{N} \sum_{k=1}^{\infty} \delta_{k, \Delta(i, j)}\left(\iint_{[0, t] \times \mathbb{R}_{0}}\left(\delta_{j i} \zeta\right) \nu_{i}(d \zeta) d s\right) \mathcal{K}_{\epsilon_{k}} h_{i} \\
& =\sum_{k=1}^{\infty} \delta_{k, \Delta(i, j)} h_{i}\left(\iint_{[0, t] \times \mathbb{R}_{0}}\left(\delta_{j i} \zeta\right) \nu_{i}(d \zeta) d s\right) \mathcal{K}_{\epsilon_{k}}=\sum_{k=1}^{\infty} \theta_{k}(t) \mathcal{K}_{\epsilon_{k}}
\end{aligned}
$$

where $\theta_{k}(t):=\delta_{k, \Delta(i, j)}\left(\iint_{[0, t] \times \mathbb{R}_{0}}\left(\delta_{j i} \zeta\right) \nu_{i}(d \zeta) d s\right) h_{i} \in H$. Because of condition (3.5) it is clear that the sum in (3.6) converges in $L^{2}\left(H, \mu_{H}\right)$ to $L(t)$.

Example 3.6. (weak Lévy process) If condition (3.5) is not fulfilled then our sequence $\left(\nu_{i}\right)_{i=1}^{N}$ does not correspond to an $H$-valued Lévy process and the formal sum in (3.6) does not converge in $L^{2}\left(H, \mu_{H}\right)$ any more. But it converges in $S(H)_{-0}$ as will follow from the below calculations. However, we don't want to consider $L(t)$ as defined in (3.6) but a "normalization" of it. More precisely, we define the weak Lévy process corresponding to our family $\left(\nu_{i}\right)_{i=1}^{N}$ of Lévy measures (fulfilling condition (3.5) or not) to be the formal sum

$$
W(t):=\sum_{i=1}^{N} W_{i}(t) h_{i}=\sum_{k=1}^{\infty} \psi_{k}(t) \mathcal{K}_{\epsilon_{k}}
$$


where $W_{i}(t):=\frac{1}{\sqrt{\lambda_{i}}} L_{i}(t)$ and

$$
\psi_{k}(t):=\delta_{k, \Delta(i, j)} \frac{1}{\sqrt{\lambda_{i}}}\left(\iint_{[0, t] \times \mathbb{R}_{0}}\left(\delta_{j i} \zeta\right) \nu_{i}(d \zeta) d s\right) h_{i} \in H .
$$

Note that $W(t)$ is a normalization of $L(t)$ in the sense that $E\left[W_{i}(t)^{2}\right]=1$ for all $i$. $W(t)$ is not in $L^{2}\left(H, \mu_{H}\right)$ but a generalized process with values in $S(H)_{-0}$ as can be seen from

$$
\begin{aligned}
\sum_{k=1}^{\infty} \epsilon_{k} !\left\|\psi_{k}(t)\right\|_{H}^{2}(2 k)^{-q} & =\sum_{k=1}^{\infty} \delta_{k, \Delta(i, j)} \frac{1}{\lambda_{i}}\left(\iint_{[0, t] \times \mathbb{R}_{0}}\left(\delta_{j i} \zeta\right) \nu_{i}(d \zeta) d s\right)^{2}(2 k)^{-q} \\
& \leq \sum_{k=1}^{\infty}(2 k)^{-q}<\infty
\end{aligned}
$$

for all $q \geq 2$. It is the weak Lévy process $W(t)$ and not the Lévy process $L(t)$ which will be the key underlying driving process in our study of stochastic differential equations. However, if $\left(\nu_{i}\right)_{i=1}^{N}$ is such that $L(t)$ exists the information contained in the integral w.r.t. $W(t)$ is identical to the information contained in the integral w.r.t. $L(t)$, but the scheme to construct the Hitsuda-Skorohod integral through the Wick product gets standardized for all families $\left(\nu_{i}\right)_{i=1}^{N}$ by considering $W(t)$ (see Section 3.4).

Example 3.7. (singular white noise) The singular white noise process $\dot{W}(t)$ is defined as the time derivative of the weak Lévy process. It is given by the following chaos expansion

$$
\dot{W}(t):=\sum_{k=1}^{\infty} \delta_{k, \Delta(i, j)} \frac{1}{\sqrt{\lambda_{i}}}\left(\int_{\mathbb{R}_{0}}\left(\delta_{j i}(t, \zeta) \zeta\right) \nu_{i}(d \zeta)\right) h_{i} \mathcal{K}_{\epsilon_{k}}=\sum_{k=1}^{\infty} \kappa_{k}(t) \mathcal{K}_{\epsilon_{k}}
$$

where $\kappa_{k}(t):=\delta_{k, \Delta(i, j)} \frac{1}{\sqrt{\lambda_{i}}}\left(\int_{\mathbb{R}_{0}}\left(\delta_{j i}(t, \zeta) \zeta\right) \nu_{i}(d \zeta)\right) h_{i} \in H$. Similarly to the weak Lévy process one can show that $\dot{W}(t) \in S(H)_{-0}$. To this purpose one uses the fact that by the form of the basis $\delta_{j i}(t, \zeta)$ (see (2.7) ) and the uniform boundedness of the Hermite functions $\xi(t)_{l}$ one has $\left\|\kappa_{k}(t)\right\|_{H} \leq C$ for some constant $C$.

3.3. Wick product. In the finite dimensional case the Wick product, denoted by $\diamond$, can be defined between $S\left(\mathbb{R}^{m \times n}\right)_{-1}$-valued and $S\left(\mathbb{R}^{n}\right)_{-1}$-valued stochastic distribution, i.e. the Wick product is an operation between generalized $\mathcal{L}\left(\mathbb{R}^{n}, \mathbb{R}^{m}\right)$-valued and generalized $\mathbb{R}^{n}$-valued random variables:

$$
\diamond: S\left(\mathbb{R}^{m \times n}\right)_{-1} \times S\left(\mathbb{R}^{n}\right)_{-1} \longrightarrow S\left(\mathbb{R}^{m}\right)_{-1} .
$$

Here $\mathcal{L}\left(\mathbb{R}^{n}, \mathbb{R}^{m}\right)$ denotes the space of linear operators between $\mathbb{R}^{n}$ and $\mathbb{R}^{m}$, which equipped with the Hilbert-Schmidt norm is identified with the Hilbert space $\mathbb{R}^{m \times n}$ in the construction of $S\left(\mathbb{R}^{m \times n}\right)_{-1}$.

In this Section we want to extend this concept to infinite dimensional Hilbert spaces. Let $U$ be another separable real Hilbert space with orthonormal basis $\left\{u_{i}\right\}_{i=1}^{M}$, where $M \in\{\mathbb{N}, \infty\}$. The Wick product would thus be an operation between a generalized random operator from $H$ to $U$ and a generalized $H$-valued random variable. However, the space $\mathcal{L}(H, U)$ of bounded linear operators between $H$ and $U$ is not appropriate to construct generalized random operators $S(\mathcal{L}(H, U))_{-1}$ because $\mathcal{L}(H, U)$ is not a Hilbert space anymore when $H$ or $U$ are infinite dimensional. Instead we will consider the space of Hilbert-Schmidt operators from $H$ to $U$ denoted by $\mathcal{L}_{2}(H, U)$. An operator $B \in \mathcal{L}(H, U)$ is Hilbert-Schmidt iff

$$
\sum_{i=1}^{N}\left\|B h_{i}\right\|_{U}<\infty
$$


The reason why $\mathcal{L}_{2}(H, U)$ is the appropriate space to take is twofold. Firstly, $\mathcal{L}_{2}(H, U)$ is a separable Hilbert space with orthonormal basis given through the double sequence $\left\{u_{j} \otimes h_{i}\right\}_{i, j}$, where $u_{j} \otimes h_{i}$ is the linear operator defined by

$$
\left(u_{j} \otimes h_{i}\right)(x):=u_{j}\left\langle h_{i}, x\right\rangle, x \in H .
$$

We can thus employ the machinery developed in the previous Section to construct spaces of $\mathcal{L}_{2}(H, U)$-valued stochastic test functions $S\left(\mathcal{L}_{2}(H, U)\right)_{\rho}$ and stochastic distributions $S\left(\mathcal{L}_{2}(H, U)\right)_{-}$. The second reason is that the relation between Wick product and Skorohod/Itô integration known from the finite dimensional case (see next Subsection) should also be valid in infinite dimensions. And as has been shown in [vG1] for the case of Itô integration w.r.t. a weak Wiener process, $\mathcal{L}_{2}(H, U)$ is the appropriate space for integrands to take values in. The same makes sense for integration w.r.t. a weak Lévy process as we will see.

Note that for $B(\omega) \in S\left(\mathcal{L}_{2}(H, U)\right)_{-\rho}$ we get the representation

$$
B(\omega)=\sum_{j, i=1}^{M, N} \sum_{\alpha \in \mathcal{J}} b_{j i} \mathcal{K}_{\alpha}(\omega)\left(u_{j} \otimes h_{i}\right)=\sum_{\alpha \in \mathcal{J}} b_{\alpha} \mathcal{K}_{\alpha}(\omega),
$$

where $b_{\alpha}=\sum_{j, i=1}^{M, N} b_{j i} \alpha\left(u_{j} \otimes h_{i}\right) \in \mathcal{L}_{2}(H, U)$.

Definition 3.8. The Wick product $B \diamond F$ of

$$
B=\sum_{\alpha \in \mathcal{J}} b_{\alpha} \mathcal{K}_{\alpha} \in S\left(\mathcal{L}_{2}(H, U)\right)_{-1}, F=\sum_{\alpha \in \mathcal{J}} c_{\alpha} \mathcal{K}_{\alpha} \in S(H)_{-1},
$$

with $b_{\alpha} \in \mathcal{L}_{2}(H, U), c_{\alpha} \in H$ is defined as

$$
B \diamond F:=\sum_{\alpha, \beta \in \mathcal{J}} b_{\alpha}\left(c_{\beta}\right) \mathcal{K}_{\alpha+\beta}=\sum_{\gamma \in \mathcal{J}}\left(\sum_{\alpha+\beta=\gamma} b_{\alpha}\left(c_{\beta}\right)\right) \mathcal{K}_{\gamma},
$$

where $b_{\alpha}\left(c_{\beta}\right)$ denotes the operator $b_{\alpha}$ applied on $c_{\alpha}$.

Example 3.9. If $B \in \mathcal{L}_{2}(H, U)$ is deterministic and $F(\omega) \in L^{2}\left(H, \mu_{H}\right)$ then

$$
B \diamond F(\omega)=B(F(\omega)) .
$$

This is easily seen from the definition.

As in the finite dimensional case, test function and distribution spaces are invariant under the Wick operation in the following sense:

Lemma 3.10. For $\rho \in\{0,1\}$ we have

$$
\begin{aligned}
B & \in S\left(\mathcal{L}_{2}(H, U)\right)_{-\rho}, F \in S(H)_{-\rho} \Longrightarrow B \diamond F \in S(U)_{-\rho}, \\
b & \in S\left(\mathcal{L}_{2}(H, U)\right)_{\rho}, f \in S(H)_{\rho} \Longrightarrow b \diamond f \in S(U)_{\rho} .
\end{aligned}
$$

Proof. We only show the statement for $\rho=-1$, the other cases being similar. There exist $q_{1}, q_{2} \in \mathbb{N}$ such that

$$
\begin{aligned}
\|B\|_{-1,-q_{1}}^{2} & =\sum_{\alpha \in \mathcal{J}}\left\|c_{\alpha}\right\|_{H}^{2}(2 \mathbb{N})^{-q_{1} \alpha}<\infty, \\
\|F\|_{-1,-q_{2}}^{2} & =\sum_{\alpha \in \mathcal{J}}\left\|b_{\alpha}\right\|_{\mathcal{L}_{2}(H, U)}^{2}(2 \mathbb{N})^{-q_{2} \alpha}<\infty .
\end{aligned}
$$

Then with $q=q_{1}+q_{2}+q_{3}$, where $q_{3}>1$,

$$
\|B \diamond F\|_{-1,-q}^{2}
$$




$$
\begin{aligned}
& =\sum_{\gamma \in \mathcal{J}}\left\|\sum_{\alpha+\beta=\gamma} b_{\alpha}\left(c_{\beta}\right)\right\|_{U}^{2}(2 \mathbb{N})^{-q \gamma} \leq \sum_{\gamma \in \mathcal{J}}\left(\sum_{\alpha+\beta=\gamma}\left\|b_{\alpha}\left(c_{\beta}\right)\right\|_{U}\right)^{2}(2 \mathbb{N})^{-q \gamma} \\
& \leq \sum_{\gamma \in \mathcal{J}}(2 \mathbb{N})^{-q_{3} \gamma}\left(\sum_{\alpha+\beta=\gamma}\left\|b_{\alpha}\right\|_{\mathcal{L}_{2}(H, U)}^{2}(2 \mathbb{N})^{-q_{2} \gamma}\right)\left(\sum_{\alpha+\beta=\gamma}\left\|c_{\beta}\right\|_{H}^{2}(2 \mathbb{N})^{-q_{1} \gamma}\right) \\
& \leq \sum_{\gamma \in \mathcal{J}}(2 \mathbb{N})^{-q_{3} \gamma}\left(\sum_{\alpha \in \mathcal{J}}\left\|b_{\alpha}\right\|_{\mathcal{L}_{2}(H, U)}^{2}(2 \mathbb{N})^{-q_{2} \alpha}\right)\left(\sum_{\beta \in \mathcal{J}}\left\|c_{\beta}\right\|_{H}^{2}(2 \mathbb{N})^{-q_{1} \beta}\right)<\infty
\end{aligned}
$$

Here we have used that $\sum_{\gamma \in \mathcal{J}}(2 \mathbb{N})^{-q_{3} \gamma}<\infty$ for $q_{3}>1$ (see $[\mathrm{Z}]$ ).

Next, we introduce the Wick composition of stochastic operator valued distributions. Let $V$ be a third separable Hilbert space.

Definition 3.11. Let $B \in S\left(\mathcal{L}_{2}(H, U)\right)_{-1}$ and $G \in S\left(\mathcal{L}_{2}(U, V)\right)_{-1}$. We then define $G \diamond B: S(H)_{-1} \longrightarrow S(V)_{-1}$ by

$$
(G \diamond B) \diamond F:=G \diamond(B \diamond F), \quad F \in S(H)_{-1}
$$

and call $G \diamond B$ for Wick composition between $G$ and $B$.

Note that because of Lemma 3.10 this definition is well defined. Also, the subspaces specified in Lemma 3.10 stay invariant under $B \diamond G$.

Example 3.12. Let $B \in S\left(L_{2}(H, H)\right)_{-1}$. Then we define the Wick exponential of $B$ as

$$
\exp ^{\diamond} B:=\sum_{n=0}^{\infty} \frac{1}{n !} B^{\diamond n}
$$

where $B^{\diamond n}:=B \triangleright B \ldots \diamond B$ is the $n$-fold Wick composition of $B$. It will become clear in Example 3.21 that $\exp ^{\S} B$ is well defined in the sense that

$$
\sum_{n=0}^{l} \frac{1}{n !} B^{\diamond n} \diamond F
$$

converges in $S(H)_{-1}$ when $l \rightarrow \infty$ for every $F \in S(H)_{-1}$.

3.4. Hitsuda-Skorohod integration. One of the most important properties of the Wick product in finite dimensions is that it enables to express Itô-Skorohod integration as a Bochner integral in $S\left(\mathbb{R}^{n}\right)_{-0}$ (for more information see [HØUZ] for the Gaussian and $[\mathrm{D} \emptyset \mathrm{P}]$ for the Lévy process case). In this Subsection we extend this concept to the infinite dimensional case.

Definition 3.13. A process $F(t): \mathbb{R} \rightarrow S(H)_{-0}$ is said to be Pettis integrable if

$$
\langle F(t), f\rangle \in L^{1}(\mathbb{R}, d t)
$$

for all $f \in S(H)_{0}$. Then the Pettis integral of $F(t)$, denoted by $\int_{\mathbb{R}} F(t) d t$, is the unique element in $S(H)_{-0}$ such that

$$
\left\langle\int_{\mathbb{R}} F(t) d t, f\right\rangle=\int_{\mathbb{R}}\langle F(t), f\rangle d t .
$$

The existence of the Pettis integral $\int_{\mathbb{R}} F(t) d t$ in $S(H)_{-0}$ follows from the fact that

$$
\int_{\mathbb{R}}\langle F(t), \cdot\rangle d t
$$


is a bounded linear operator on $S(H)_{0}$. If we represent $F(t) \in S(H)_{-0}$ as $F(t)=$ $\sum_{\alpha \in \mathcal{J}} c_{\alpha}(t) \mathcal{K}_{\alpha}$ then it is straight forward to show (proof analogous to [FS]) that $F(t)$ is Pettis integrable if

$$
\sum_{\alpha \in \mathcal{J}} \alpha !\left(\int_{\mathbb{R}}\left\|c_{\alpha}(t)\right\|_{H} d t\right)^{2}(2 \mathbb{N})^{-q \alpha}<\infty
$$

for some $q \in \mathbb{N}$. In this case we have

$$
\int_{\mathbb{R}} F(t) d t=\sum_{\alpha \in \mathcal{J}} \int_{\mathbb{R}} c_{\alpha}(t) d t \mathcal{K}_{\alpha}
$$

where $\int_{\mathbb{R}} c_{\alpha}(t) d t$ is a Bochner integral in $H$.

Using the Wick product defined in the previous subsection we now construct the abstract Hitsuda-Skorohod integral w.r.t. to a weak Lévy process. Let again $U$ be another separable real Hilbert space with orthonormal basis $\left\{u_{i}\right\}_{i=1}^{M}, M \in\{\mathbb{N}, \infty\}$, and recall that $\dot{W}(t)$ denotes the singular white noise defined in example 3.7.

Definition 3.14. Suppose $B(t): \mathbb{R} \rightarrow S\left(\mathcal{L}_{2}(H, U)\right)_{-0}$ is such that $B(t) \diamond \dot{W}(t)$ is Pettis integrable in $S(U)_{-0}$. Then the abstract Hitsuda-Skorohod integral w.r.t. to the weak Lévy process $W(t)$ is defined as

$$
\int_{\mathbb{R}} B(t) \diamond \dot{W}(t) d t \in S(U)_{-0} .
$$

The following Proposition gives a sufficient criteria for the existence of the abstract Hitsuda-Skorohod integral.

Proposition 3.15. Let $B(t)=\sum_{\alpha \in \mathcal{J}} b_{\alpha}(t) \mathcal{K}_{\alpha} \in S\left(\mathcal{L}_{2}(H, U)\right)_{-0}$. If

$$
\sup _{\alpha \in \mathcal{J}}\left\{\alpha !(2 \mathbb{N})^{-q \alpha} \int_{\mathbb{R}}\left\|b_{\alpha}(t)\right\|_{\mathcal{L}_{2}(H, U)}^{2} d t\right\}<\infty
$$

for some $q \in \mathbb{N}$, then the abstract Hitsuda-Skorohod integral of $B(t)$ exists.

Proof. By the definition of the singular white noise we have

$$
B(t) \diamond \dot{W}(t)=\sum_{\gamma \in \mathcal{J}}\left(\sum_{\alpha+\epsilon_{k}=\gamma} b_{\alpha}(t)\left(\kappa_{k}(t)\right)\right) \mathcal{K}_{\gamma}=: \sum_{\gamma \in \mathcal{J}} p_{\gamma}(t) \mathcal{K}_{\gamma} .
$$

We first consider

$$
\begin{aligned}
\left(\int_{\mathbb{R}}\left\|p_{\gamma}(t)\right\|_{U} d t\right)^{2} & \leq \int_{\mathbb{R}}\left\|p_{\gamma}(t)\right\|_{U}^{2} d t \\
& \leq \int_{\mathbb{R}}\left(\sum_{\alpha+\epsilon_{k}=\gamma}\left\|b_{\alpha}(t)\right\|_{\mathcal{L}_{2}(H, U)}\left\|\kappa_{k}(t)\right\|_{H}\right)^{2} d t \\
& \leq C \sum_{\alpha+\epsilon_{k}=\gamma} \int_{\mathbb{R}}\left\|b_{\alpha}(t)\right\|_{\mathcal{L}_{2}(H, U)}^{2} d t
\end{aligned}
$$

where we used the boundedness of $\left\|\kappa_{k}(t)\right\|_{H}$ as in example 3.7. Taking into account that $\left(\alpha+\epsilon_{k}\right) ! \leq \alpha !(|\alpha|+1)$, we can now verify condition (3.9) for $q>\frac{1}{2}$.

We conclude this subsection by demonstrating that the abstract Hitsuda-Skorohod integral also in infinite dimensions extends the Itô type integral w.r.t. a Hilbert space valued Lévy process. Literature about stochastic integration w.r.t. infinite dimensional Hilbert space valued Lévy process seems to be rather scarce. Two references where such integrals are constructed are $[\mathrm{C}-\mathrm{M}]$ and $[\mathrm{vG} 2]$, whereas in $[\mathrm{K}]$ and $[\mathrm{MPe}]$ stochastic integration w.r.t. general martingales in Hilbert spaces is treated. We will here illustrate that the abstract Hitsuda-Skorohod integral generalizes the integral based on a series approach introduced 
in [vG2] (which in turn in [vG2] was shown to be equivalent to the integral in [C-M]). Let $L(t)$ be an $H$-valued Lévy process as defined in example 3.5 with corresponding independent scalar decomposition $L_{i}(t)$ and with covariance operator $Q$ with eigenvalues $\lambda_{i}$. Denote by $\left\{\mathcal{F}_{t}\right\}_{t>0}, \mathcal{F}_{t} \subset \mathcal{F}_{H}$, the augmented filtration generated by $L(t)$. Let a stochastic operator valued process

$$
\Phi(t):[0, T] \rightarrow L^{2}\left(\mathcal{L}(H, U), \mu_{H}\right)
$$

be predictable and piecewise continuous in $t$, where $\mathcal{L}(H, U)$ is equipped with the operator norm, and let $\Phi_{i}(t):=\Phi(t)\left(h_{i}\right)$. Then the stochastic integral of $\Phi(t)$ w.r.t. $L(t)$ is in [vG2] defined as the following $L^{2}\left(U, \mu_{H}\right)$-limit (which always exists)

$$
\int_{0}^{T} \Phi(t) d L(t):=\lim _{L^{2}\left(U, \mu_{H}\right)}^{n} \sum_{i=1}^{N} \sum_{k=0}^{n-1} \Phi_{i}\left(t_{k}\right)\left(L_{i}\left(t_{k+1}\right)-L_{i}\left(t_{k}\right)\right)
$$

for a sequence of refining partitions $0=t_{0}<\ldots<t_{n}=T$.

Proposition 3.16. Define the stochastic $\mathcal{L}(H, U)$-valued process $B(t)$ through

$$
B(t)\left(h_{i}\right):=\sqrt{\lambda_{i}} \Phi_{i}(t),
$$

where $\lambda_{i}$ and $\Phi_{i}(t)$ are as above. Then $B(t) \in L^{2}\left(\mathcal{L}_{2}(H, U), \mu_{H}\right)$ for all $t$, its abstract Hitsuda-Skorohod integral exists and

$$
\int_{0}^{T} B(t) \diamond \dot{W}(t) d t=\int_{0}^{T} \Phi(t) d L(t) .
$$

Proof. It is sufficient to show the statement for a simple process $\Phi(t)$ of the form

$$
\Phi(t)=\Phi 1_{(a, b]}(t),
$$

where $0 \leq a<b \leq T$ and $\Phi \in L^{2}\left(\mathcal{L}(H, U), \mu_{H}\right)$ is $\mathcal{F}_{a \text {-adapted. }}$ -

Note first that

$$
\|B(t)\|_{\mathcal{L}_{2}(H, U)}=\sum_{i=1}^{N} \lambda_{i}\left\|\Phi_{i}(t)\right\|_{U} \leq\|\Phi(t)\|_{\mathcal{L}(H, U)} \sum_{i=1}^{N} \lambda_{i}<\infty,
$$

and we get $B(t) \in L^{2}\left(\mathcal{L}_{2}(H, U), \mu_{H}\right)$. It is then straight forward to see that condition (3.10) is fulfilled and the Hitsuda-Skorohod integral of $B(t)$ exists.

To verify equality (3.11) we represent $B(t)$ and $\dot{W}(t)$ in the following way

$$
\begin{aligned}
B(t) & =\sum_{j, i=1}^{M, N} \sum_{\alpha \in \mathcal{J}} b_{j i}(t) \mathcal{K}_{\alpha}(\omega)\left(u_{j} \otimes h_{i}\right)=\sum_{j, i=1}^{M, N} B_{j i}(t)\left(u_{j} \otimes h_{i}\right), \\
\dot{W}(t) & =\sum_{i=1}^{N} \sum_{k \in \Delta(i, \cdot)} \kappa_{i k}(t) \mathcal{K}_{\epsilon_{k}} h_{i}=\sum_{i=1}^{N} \dot{W}_{i}(t) h_{i},
\end{aligned}
$$

where $B_{j i}(t) \in L^{2}\left(\mathbb{R}, \mu_{H}\right)$ is predictable, $\kappa_{i k}(t)=\left\langle\kappa_{k}(t), h_{i}\right\rangle_{H}$ and $\dot{W}_{i}(t)$ is the chaos expansion of the scalar white noise corresponding to the scalar Lévy process $W_{i}(t)$. We then get

$$
\begin{aligned}
& B(t) \diamond \dot{W}(t)=\sum_{\alpha, k} \sum_{i=1}^{N} \sum_{j=1}^{M} b_{j i}(t) \kappa_{i k}(t) u_{j} \mathcal{K}_{\alpha+\epsilon_{k}} \\
= & \sum_{i=1}^{N} \sum_{j=1}^{M} \sum_{\alpha, k \in \Delta(i, \cdot)} b_{j i}(t) \kappa_{i k}(t) \mathcal{K}_{\alpha+\epsilon_{k}} u_{j} \stackrel{*}{=} \sum_{i=1}^{N} \sum_{j=1}^{M} \int_{a}^{b} B_{j i}(t) d W_{i}(t) u_{j} \\
= & \sum_{i=1}^{N} \sum_{j=1}^{M} B_{j i}(a) u_{j} \int_{a}^{b} d W_{i}(t)=\sum_{i=1}^{N} \sqrt{\lambda_{i}} \Phi_{i}(a) \int_{a}^{b} d W_{i}(t)
\end{aligned}
$$




$$
=\sum_{i=1}^{N} \Phi_{i}(a)(L(b)-L(a))=\int_{0}^{T} \Phi(t) d L(t) .
$$

where $\int_{a}^{b} B_{j i}(t) d W_{i}(t)$ is the usual Itô integral and where we have used the known relation between the abstract Hitsuda-Skorohod integral and the Itô integral in one dimension in the equality marked by $*$.

Remark 3.17. As can be seen from Proposition 3.16, we have to "scale" the operator process $\Phi(t)$ in the Itô integral by the sequence $\left\{\sqrt{\lambda_{i}}\right\}_{i}$ in order to get the corresponding operator in the Hitsuda-Skorohod integral. This is due to the fact that we integrate w.r.t. the "normalized" weak Lévy process $W(t)$ in the Hitsuda-Skorohod integral; i.e. we standardize the covariance information of the integrator and transfer this information into the integrand. In this way, the space of Hilbert-Schmidt operators $L_{2}(H, U)$ is the optimal space for the integrands in the Hitsuda-Skorohod integral to take values in for all sequences of Lévy measures $\left(\nu_{i}(d \zeta)\right)_{i=1}^{N}$.

If we didn't consider the weak weak Lévy process $W(t)$ but the Lévy process $L(t)$ corresponding to $\left(\nu_{i}(d \zeta)\right)_{i=1}^{N}$ as integrator, the alternative but less convenient approach would be to adopt the corresponding Hilbert spaces. More precisely, like in the construction of the stochastic integral w.r.t. a $Q$-Brownian motion (see f.ex. [dPZ]), consider the Hilbert space $H_{0}=Q(H) \subset H$ with inner product induced by $Q$ (as before $Q$ is the covariance operator of $L(t))$. Then $L(t)$ would be in $S\left(H_{0}\right)_{-0}$ with the same chaos expansion as the one of $W(t)$ in $S(H)_{-0}$. The abstract Hitsuda-Skorohod integral w.r.t. $L(t)$, which then generalizes the Itô integral without scaling the integrands, would be defined for integrands taking values $S\left(L_{2}\left(H_{0}, U\right)\right)_{-0}$.

3.5. Hermite transform. The last white noise concept we treat in this Section is the Hermite transform. As in the finite dimensional case introduced in [HØUZ], the Hermite transform is a very useful tool to characterize the elements and the topology of $S(H)_{-1}$. In the following of this Subsection we only state the key results we need later on. The proofs are analogue to the ones in [FS] where the Hermite transform and its properties for infinite dimensional Hilbert spaces in the Gaussian case is elaborated.

We denote by $H_{\mathbb{C}}$ resp. $U_{\mathbb{C}}$ the complexification of $H$ resp. $U$. Further, we set $z^{\alpha}=$ $\prod_{i} z_{i}^{\alpha_{i}}$ for $z \in \mathbb{C}^{\mathbb{N}}$ and a multiindex $\alpha$.

Definition 3.18. The Hermite transform of $F=\sum_{\alpha \in \mathcal{J}} c_{\alpha} \mathcal{K}_{\alpha}(\omega) \in S(H)_{-1}$ is defined as

$$
\mathcal{H} F(z):=\sum_{\alpha \in \mathcal{J}} c_{\alpha} z^{\alpha}
$$

for $z \in \mathbb{C}^{\mathbb{N}}$ so that the limit exists in $H_{\mathbb{C}}$.

In the following we often drop the dependence on $z$ and only write $\mathcal{H} F$. From the definition it becomes clear that the Hermite transform carries Wick operations over to deterministic operations. More precisely, for $B \in S\left(\mathcal{L}_{2}(H, U)\right)_{-1}, F \in S(H)_{-1}$ we have

$$
\mathcal{H}(B \diamond F)=\mathcal{H} B(\mathcal{H} F)
$$

where $\mathcal{H} B(z) \in \mathcal{L}_{2}\left(H_{\mathbb{C}}, U_{\mathbb{C}}\right)$ and $\mathcal{H} F(z) \in H_{\mathbb{C}}$. Further, for $B \in S\left(\mathcal{L}_{2}(H, U)\right)_{-1}, G \in$ $S\left(\mathcal{L}_{2}(U, V)\right)_{-1}$

$$
\mathcal{H}(G \diamond B)=\mathcal{H} G \mathcal{H} B
$$

where $\mathcal{H} G \mathcal{H} B$ denotes the composition of the operators $\mathcal{H} B(z) \in \mathcal{L}_{2}\left(U_{\mathbb{C}}, V_{\mathbb{C}}\right)$ and $\mathcal{H} B(z) \in$ $\mathcal{L}_{2}\left(H_{\mathbb{C}}, U_{\mathbb{C}}\right)$.

For $\mathbb{N} \ni q>1$ we define the following infinite dimensional neighborhood of 0 in $\mathbb{C}^{\mathbb{N}}$ :

$$
\mathbb{K}_{q}:=\left\{z \in \mathbb{C}^{\mathbb{N}}:\left|z_{i}\right|<(2 i)^{-q}, i \in \mathbb{N}\right\} .
$$

The Hermite transform characterizes stochastic distributions in $S(H)_{-1}$ : 
Proposition 3.19. 1) For $F \in S(H)_{-1}$ there exists $q>1$ such that $\mathcal{H} F(z)$ converges absolutely for all $z \in \mathbb{K}_{q}$ and $\mathcal{H} F(z)$ is bounded on $\mathbb{K}_{q}$.

2) Conversely, suppose $g(z)=\sum_{\alpha \in \mathcal{J}} c_{\alpha} z^{\alpha}$ converges absolutely and is bounded on $\mathbb{K}_{q}$ for some $q>1$. Then there exists a unique $F \in S(H)_{-1}$ such that $\mathcal{H} F(z)=g(z)$, namely

$$
F=\sum_{\alpha \in \mathcal{J}} c_{\alpha} \mathcal{K}_{\alpha}(\omega)
$$

Also, the convergence of sequences in $S(H)_{-1}$ can be connected to the convergence of the corresponding Hermite transforms:

Proposition 3.20. The following statements are equivalent:

1) $F_{n} \longrightarrow F$ in the topology of $S(H)_{-1}$.

2) There exists $q>1$ such that $\mathcal{H} F_{n}(z) \longrightarrow \mathcal{H} F(z)$ converges pointwise and boundedly on $\mathbb{K}_{q}$.

Example 3.21. Let $B \in S\left(L_{2}(H, H)\right)_{-1}$ and $F \in S(H)_{-1}$ We then consider the sum $\sum_{n=0}^{l} \frac{1}{n !} B^{\diamond n} \diamond F$. Applying the Hermite transform yields

$$
\sum_{n=0}^{l} \frac{1}{n !}(\mathcal{H} B)^{n}(\mathcal{H} F)
$$

However, because $\mathcal{H} B$ is a Hilbert-Schmidt operator on $H_{\mathbb{C}}$ the sum in (3.14) converges pointwise boundedly on some $K_{q}$ to $e^{\mathcal{H B}}(\mathcal{H} F)$. So by Proposition 3.20 the Wick exponential in Example 3.12 is a well defined concept.

As a consequence of Proposition 3.20 we can characterize differentiation and integration in $S(H)_{-1}$ via the corresponding Hermite transforms.

Theorem 3.22. Consider two processes $X(t), F(t):[a, b] \longrightarrow S(H)_{-1}$. The following statements are equivalent:

1) $X(t)$ is differentiable in the topology of $S(H)_{-1}$ on $[a, b], F(t)$ is continuous in the topology of $S(H)_{-1}$ on $[a, b]$, and

$$
\frac{d X(t)}{d t}=F(t)
$$

2) There exists $q>1$ such that

a: $H X(t, z)$ and $H F(t, z)$ exist for all $z \in K_{q}, t \in[a, b]$.

b: $\operatorname{HF}(t, z)$ is continuous on $[a, b]$ and bounded on $[a, b] \times K_{q}$.

c: $H X(t, z)$ is differentiable on $[a, b]$ with

$$
\frac{d \mathcal{H} X(t, z)}{d t}=\mathcal{H} F(t, z)
$$

for all $(t, z) \in[a, b] \times K_{q}$.

Example 3.23. Let us show that in $S(H)_{-1}$ the singular white noise $\dot{W}(t)$ is differentiable. We have that for $q>2$

$$
\mathcal{H} \dot{W}(t)=\sum_{k=1}^{\infty} \kappa_{k}(t) z_{k}
$$

converges for $z \in K_{q}$. Because

$$
\sup _{t \in[a, b]} \xi_{j}^{\prime}(t) \leq C j
$$

where the constant $C$ only depends on $[a, b]$ we get that $\left\|\kappa_{k}^{\prime}(t)\right\|_{H}^{2} \leq C^{2} i(k)^{2} \lambda_{j(k)} \leq C_{1} k^{2}$ for another constant $C_{1}$. Hence

$$
\left|\sum_{k=1}^{\infty} \kappa_{k}^{\prime}(t) z_{k}\right|^{2} \leq\left(\sum_{k=1}^{\infty}\left\|\kappa_{k}^{\prime}(t)\right\|_{H}^{2}(2 \mathbb{N})^{-4 \epsilon_{k}}\right)\left(\sum_{k=1}^{\infty}\left|z_{k}\right|^{2}(2 \mathbb{N})^{4 \epsilon_{k}}\right)
$$




$$
\leq C_{1}\left(\sum_{k=1}^{\infty} k^{2}(2 k)^{-4}\right)\left(\sum_{\alpha \in \mathcal{J}}\left|z^{\alpha}\right|^{2}(2 \mathbb{N})^{4 \alpha}\right)<C_{3}
$$

on $(t, z) \in[a, b] \times K_{4}$. Because clearly $\sum_{k=1}^{\infty} \kappa_{k}^{\prime}(t) z_{k}$ is continuous in $t$ we get by Theorem 3.22 that $\dot{W}(t)$ is differentiable in $S(H)_{-1}$ with

$$
\frac{d \dot{W}(t)}{d t}=\sum_{k=1}^{\infty} \kappa_{k}^{\prime}(t) \mathcal{K}_{k}
$$

Similarly one can show that the weak Lévy process $W(t)$ is differentiable in $S(H)_{-1}$ with derivative $\dot{W}(t)$, and that further $\dot{W}(t)$ is infinitely many times differentiable.

Theorem 3.24. If $X(t):[a, b] \longrightarrow S(H)_{-1}$ is such that $\int_{a}^{b} \mathcal{H} X(t, z) d t<\infty$ on $\mathbb{K}_{q}$ for some $q>1$ and

$$
\sup _{(t, z) \in[a, b] \times K_{q}}\|\mathcal{H} X(t, z)\|_{H_{\mathbb{C}}}<\infty
$$

then $\int_{a}^{b} X(t) d t$ exists in $S(H)_{-1}$ and

$$
\mathcal{H} \int_{a}^{b} X(t) d t=\int_{a}^{b} \mathcal{H} X(t, z) d t .
$$

\section{Stochastic Differential Equations in $S(H)_{-1}$}

We now want to apply the machinery developed in the previous Section to stochastic differential equations with values in spaces of stochastic distributions. Hilbert space valued differential equations often include unbounded linear operators and we first discuss the concept of these in the framework of stochastic distribution. Let $H, U$ be separable real Hilbert spaces as before.

4.1. Deterministic operators on $S(H)_{-1}$. Let $A: H \longrightarrow U$ be a linear possibly unbounded operator with domain $\mathcal{D}(A) \subseteq H$. We then define the action of $A$ on $S(H)_{-1}$.

Definition 4.1. The domain of $A$ in $S(H)_{-1}$ is defined to be

$$
\mathcal{D}(A)_{-1}:=\left\{F=\sum_{\alpha \in \mathcal{J}} c_{\alpha} \mathcal{H}_{\alpha} \in S(H)_{-1}: \sum_{\alpha \in \mathcal{J}}\left\|A\left(c_{\alpha}\right)\right\|_{U}^{2}(2 \mathbb{N})^{-q \alpha}<\infty\right\}
$$

for some $q \in N$. The action of $A$ on $F \in \mathcal{D}(A)_{-1}$ is set to be

$$
A(F):=\sum_{\alpha \in \mathcal{J}} A\left(c_{\alpha}\right) \mathcal{H}_{\alpha} \in S(U)_{-1} .
$$

We define analogously the action of $A$ on any $S(H)_{ \pm \rho}, \rho \in\{0,1\}$ with corresponding domains $\mathcal{D}(A)_{ \pm \rho}$.

Remark 4.2. If $A \in \mathcal{L}(H, U)$ is a bounded operator then it is immediate to see that $\mathcal{D}(A)_{ \pm \rho}=S(H)_{ \pm \rho}, \rho \in\{0,1\}$. If further $A \in \mathcal{L}_{2}(H, U)$ we get that

$$
A(F)=A \diamond F \text {. }
$$

Note that we can extend this relation for all operators $A \in \mathcal{L}(H, U)$ that are compositions of Hilbert-Schmidt operators, i.e.

$$
A=B_{1} \circ \ldots \circ B_{n}, \quad B_{1}, \ldots, B_{n} \in \mathcal{L}_{2}(H, U),
$$

with the help of the Wick composition in Definition 3.11.

Like in [FS] one can show that the Hermite transform is also factorizing unbounded operators in the desired sense as long as they are closed:

Lemma 4.3. If $A: H \longrightarrow U$ is a closed operator and $F \in \mathcal{D}(A)_{-1}$ then

$$
\mathcal{H}(A(F))(z)=A(\mathcal{H} F(z)) .
$$


4.2. Stochastic Cauchy problem. Consider the following $U$-valued stochastic differential equation with additive Lévy noise

$$
\begin{aligned}
d X_{t} & =A X_{t} d t+B d W_{t}, \\
X_{0} & =x \in \mathcal{D}(A), \quad 0 \leq t<T,
\end{aligned}
$$

where $A$ is the generator of a $C_{0}$-semigroup $S_{t}$ on $U$ (in particular $A$ is a densely defined closed operator), $W(t)$ is an $H$-valued weak Lévy process and $B \in \mathcal{L}_{2}(H, U)$ is a HilbertSchmidt operator. In the context of the theory of classical Hilbert space valued SDE's one can show like f. ex. in $[\mathrm{C}-\mathrm{M}]$ that analogously to the Gaussian case there exists a weak solution of equation (4.1) under the condition that $S_{t} B \in \mathcal{L}_{2}(H, U)$ with

$$
\int_{0}^{T}\left\|S_{t} B\right\|_{\mathcal{L}_{2}(H, U)} d t<\infty .
$$

This solution is then given by

$$
X_{t}=S_{t} x+\int_{0}^{t} S(t-s) B d W_{t}, \quad 0 \leq t<T .
$$

Interpreting the stochastic integral $B d W_{t}$ as a Hitsuda-Skorohod integral $B \diamond \dot{W}_{t} d t$ we arrive at the following reformulation of (4.1) in the white noise framework

$$
\begin{aligned}
\frac{d X_{t}}{d t} & =A X_{t}+B \diamond \dot{W}_{t}, \\
X_{0} & =x \in \mathcal{D}(A), \quad 0 \leq t<T .
\end{aligned}
$$

Using Definition 4.1 we can extend the classical result about weak solutions of (4.1) in the following way:

Theorem 4.4. Let $A$ be the generator of a strongly continuous semigroup $\left\{S_{t}\right\}_{t>0}$ on $U$ and $B \in \mathcal{L}(H, U)$ a bounded operator. Then the process

$$
X_{t}=S_{t}(x)+\int_{0}^{t} S_{t-s} B\left(\dot{W}_{s}\right) d s
$$

solves equation (4.4) uniquely in $S(U)_{-1}$.

Proof. For notational convenience we denote $\tilde{G}:=\mathcal{H} G$ for a distribution $G$ in the rest of the proof. First note that because a strongly continuous semigroup is exponentially bounded it is not difficult to see that $S_{t-s} B\left(\dot{W}_{s}\right)$ is Pettis integrable, so the integral in (4.5) is well defined. Takin the Hermite transform in (4.5) we obtain

$$
\tilde{X}_{t}=S_{t}(\tilde{x})+\int_{0}^{t} S_{t-s} B\left(\dot{\tilde{W}}_{s}\right) d s .
$$

Then the following calculations are standard from the theory of deterministic linear equations (see f.ex.[F], or [dPZ], [MFA] for a short overview). Define the integral

$$
v(s)=\int_{0}^{t-s} S_{r} B\left(\dot{\dot{W}}_{s}\right) d r
$$

and consider its derivative which by Example 3.23 is well defined

$$
v^{\prime}(s)=-S_{t-s} B\left(\tilde{\dot{W}}_{s}\right)+\int_{0}^{t-s} S_{r} B\left(\tilde{\dot{W}}_{s}^{\prime}\right) d r .
$$

Integrating in $s$ from 0 to $t$ yields

$$
\int_{0}^{t} S_{t-s} B\left(\tilde{\dot{W}}_{s}\right) d s=\int_{0}^{t} \int_{0}^{t-s} S_{r} B\left(\tilde{W}_{s}^{\prime}\right) d r d s-v(t)+v(0) .
$$


Because $v(t)=0$ we get

$$
\tilde{X}_{t}=S_{t}(\tilde{x})+\int_{0}^{t} \int_{0}^{t-s} S_{r} B\left(\tilde{W}_{s}^{\prime}\right) d r d s+\int_{0}^{t} S_{r} B\left(\tilde{\dot{W}}_{0}\right) d r .
$$

Using the formula (see $[\mathrm{F}]$ )

$$
A\left(\int_{0}^{t} S_{s} u d s\right)=S_{t}(u)-u
$$

(note that $u \in \mathcal{D}(A)$ is not required), we obtain

$$
\begin{aligned}
A\left(\tilde{X}_{t}\right) & =A S_{t}(\tilde{x})+\int_{0}^{t} S_{t-s} B\left(\tilde{\dot{W}}_{s}^{\prime}\right) d s-\int_{0}^{t} B\left(\tilde{\dot{W}}_{s}^{\prime}\right) d s+S_{t} B\left(\tilde{\dot{W}}_{0}\right)-B\left(\tilde{\dot{W}}_{0}\right) \\
& =S_{t}^{\prime}(\tilde{x})+\int_{0}^{t} S_{t-s} B\left(\tilde{\dot{W}}_{s}^{\prime}\right) d s-B\left(\tilde{W}_{t}\right)+\tilde{S}_{t} B\left(\tilde{W}_{0}\right) .
\end{aligned}
$$

Hence, differentiating (4.6) yields

$$
\frac{d \tilde{X}_{t}}{d t}=A \tilde{X}_{t}+B\left(\dot{W}_{t}\right)
$$

Now, by Theorem 3.22 we can extract the Hermite transform in equation (4.7) and the result follows.

Note that for a Pettis integrable process $F_{s}$ the process $S_{t-s} F_{s}$ is again Pettis integrable by the exponentially boundedness of $S_{s}$. So an immediate generalization of Theorem 4.4 is that

solves the equation

$$
X_{t}=S_{t}(x)+\int_{0}^{t} S_{t-s}\left(F_{s}+B\left(\dot{W}_{s}\right)\right) d s
$$

$$
\begin{aligned}
\frac{d X_{t}}{d t} & =\left[A X_{t}+F_{t}\right]+B \diamond \dot{W}_{t}, \\
X_{0} & =x \in \mathcal{D}(A), \quad 0 \leq t<T .
\end{aligned}
$$

We remark that since

$$
\sum_{k=0}^{\infty}\left\|\int_{0}^{T} S_{T-s}\left(B\left(\kappa_{k}(s)\right)\right) d s\right\|_{U}^{2} \leq C \sum_{k=0}^{\infty} \int_{0}^{T}\left\|S_{T-s}\left(B\left(h_{k}\right)\right)\right\|_{U}^{2} d s
$$

we see that, assuming $F_{s} \in U$ is Bochner integrable, $X_{t}$ is a solution in $L^{2}\left(U, \mu_{U}\right)$ if

$$
\int_{0}^{T}\left\|S_{s} B\right\|_{\mathcal{L}_{2}(H, U)}^{2} d s<\infty
$$

which was condition (4.3).

Further, the concepts developed in the previous Section allow us to consider more general equations with random coefficients being stochastic distributions of the form

$$
\begin{aligned}
\frac{d X_{t}}{d t} & =A \diamond X_{t}+F_{t}, \\
X_{0} & =x \in S(U)_{-1}, \quad 0 \leq t<T,
\end{aligned}
$$

where $A \in S\left(L_{2}(H, U)\right)_{-1}$ and $F_{t} \in S(U)_{-1}$.

Theorem 4.5. Assume that $F_{t}$ is continuous in $t$ in the topology of $S(U)_{-1}$. Then the process

$$
X_{t}=\exp ^{\S}(t A) \diamond x+\int_{0}^{t}\left(\exp ^{\S}((t-s) A) \diamond F_{s}\right) d s
$$

solves equations (4.10) uniquely in $S(U)_{-1}$. 
Proof. For notational convenience we denote $\tilde{G}:=\mathcal{H} G$ for a distribution $G$ in the rest of the proof. First note that since $\exp ^{\diamond}((t-s) A) \diamond F_{s}$ is continuous in $s$ in the topology of $S(U)_{-1}$, $\exp ^{\diamond}((t-s) A) \diamond F_{s}$ is $S(U)_{-1}$-integrable (see [FS]) and the integrand in (4.11) is well defined. Takin the Hermite transform in (4.11) yields

$$
\tilde{X}_{t}=\exp (t \tilde{A})(\tilde{x})+\int_{0}^{t} \exp ((t-s) \tilde{A})\left(\tilde{F}_{s}\right) d s .
$$

We observe that $\{\exp (t \tilde{A})\}_{t>0}$ is the strongly continuous semigroup on $U_{\mathbb{C}}$ generated by $\tilde{A}$. Clearly, since $\tilde{A}$ is a bounded operator, $\tilde{X}_{t}$ is the variation of constant formula that solves

$$
\frac{d \tilde{X}_{t}}{d t}=\tilde{A}\left(\tilde{X}_{t}\right)+\tilde{F}_{t}
$$

Now, given the nature of $X_{t}$, we can apply Theorem 3.22 and extract the Hermite transform in equation (4.12). This yields the desired result.

In particular, if $F_{t}=B_{t} \diamond \dot{W}_{t}$ for a continuous $B_{t}$, the process $X_{t}$ given in (4.11) solves a Lévy white noise driven stochastic Cauchy problem with random coefficients. However, $A$ takes values in a space of generalized bounded operators and is not allowed to be of unbounded nature in this setting.

Remark 4.6. Let us mention that the above techniques can be employed in an analogous way to treat linear stochastic differential equations with additive Lévy noise where $A$ is the generator of an $n$-times integrated semigroup. For this matter it is essential that the white noise $\dot{W}_{t}$ is infinitely many times differentiable in $S(H)_{-1}$.

Example 4.7. As a first illustration we consider the stochastic Lévy noise driven heat equation

$$
\begin{aligned}
d X(t, x) & =\triangle_{x} X(t, x) d t+d W(t, x) \\
X(t, x) & =0 \text { for } t \in[0, T], x \in \partial \mathcal{O}, \quad X(0, x)=0 \text { for } x \in \mathcal{O},
\end{aligned}
$$

where $O=\left\{x \in R^{n} ; 0<x_{k}<a_{k}, k=1, \ldots, n\right\}, n \in \mathbb{N}$. We interpret (4.13) as equation of type (4.4) with $H=L^{2}(\mathcal{O}), B=I, A=\triangle_{x}$ (in the sense of distributions) and $\mathcal{D}(A)=H^{2,2}(\mathcal{O}) \cap H_{0}^{1,2}(\mathcal{O})$. The spectrum of $A$ is given by

$$
\left\{-\sum_{i=1}^{n} \frac{k_{i}^{2} \pi^{2}}{a_{i}^{2}} ; k_{1}, \ldots, k_{n} \in \mathbb{N}^{*}\right\} .
$$

Let $\left\{-\lambda_{k}\right\}$ be an ordering of the eigenvalues with corresponding eigenvector basis $\left\{h_{k}\right\}$. Then $A$ generates a $C_{0}$ semigroup on $H$ given through

$$
S_{t}(u)=\sum_{k=1}^{\infty}\left\langle u, h_{k}\right\rangle_{H} e^{-\lambda_{k} t} h_{k}, u \in H
$$

and the solution of the heat equation with values in $S(H)_{-0}$ is given by (4.4). It is not difficult to see that

$$
\int_{0}^{T}\left\|S_{s}\right\|_{\mathcal{L}_{2}(H)}^{2} d s=\sum_{k=1}^{\infty} \frac{1}{2 \lambda_{k}}\left(1-e^{-2 \lambda_{k} T}\right) \leq \sum_{k=1}^{\infty} \frac{1}{2 \lambda_{k}} .
$$

Hence only if $n=1$ the solution of the heat equation is $H$-valued.

Acknowledgements The author thanks Bernt Øksendal and Frank Proske for valuable comments and discussions. 


\section{REFERENCES}

[AØPU] Aase, K., Øksendal, B., Privault, N., Uboe, J.:White noise generalizations of the ClarkHaussmann-Ocaone theorem with application to mathematical finance, Finance and Stochatics 4 (2000), 465-496.

[AKS] Albeverio,S., Kondratiev, Y.G., Streit L.:How to generalize White Noise Analysis to non-Gaussian spaces, Dynamics of complex and irregular systems, Ed: Ph Blanchard et al., World Scientific (1993).

[AR] Albeverio,S., Ruediger, B.: Stochastic integrations of Banach valued functions with respect to compensated Poisson random measures and the Lévy-Itô decomposition theorem, preprint, Universitaet Bonn.

[A] Alshansky, M.: Differential equations in spaces of Hilbert space valued distributions, Bul. Austral. Math. Soc., Vol. 68 (2003), pp. 491-500.

[B] Bertoin, J.: Lévy Processes. Cambridge University Press, Cambridge 1996.

[C-M] Chojnowska-Michalik, A.: On processes of Ornstein-Uhlenbeck type in Hilbert space, Stochastics, 21 (1987), pp. 251-286.

[dPZ] Da Prato, G., Zabczyk, J.: Stochastic equations in infinite dimensions, Cambridge University press, 1992.

[DØP] di Nunno G., Øksendal, B., Proske, F.:White noise analysis for Lévy processes. Journal of Funct. Ana. 206 (2004), pp.109-148.

[F] Fattorini, H.O.: The Cauchy Problem, Encycl. Math.and Appl. 18, Addison-Wesley, Reading, Mass (1983).

[FS] Filinkov, A.I.., Sorensen, J.: Differential equations in spaces of abstract stochastic distributions, Stochastics and Stochastics Reports, 2002, Vol.72 (3-4), pp.129-173.

$[\mathrm{H}] \quad$ Hida, T.: White noise analysis and its apllications, Proc. Int. Math. Conf., ed. by Chen, L:H:Y: Chen et al. North-Holland, Amsterdam, 43-48, 1982.

[HKPS] Hida, T., Kuo, H.-H., Potthoff, J., Streit, J.: White Noise. An Infinite Dimensional Approach. Kluwer (1993).

[HØUZ] Holden, H., Øksendal, B., Ubøe, J., Zhang T.-S.: Stochastic Partial Differential Equations- A Modeling, White Noise Functional Approach. Birkhäuser, Boston 1996.

[JS] Jacod, J., Shiryaev, A.N.: Limit Theorems for Stochastic Processes. Springer, Berlin Heidelberg New York (1987).

[KDSU] Kondratiev, Y.G., Da Silva, J.L., Streit L, Us, G..: Analysis on Poisson and Gamma spaces, Inf. Dim. Anal. Quant. Prob. Rel. Topics, 1 (1998), pp. 91-117.

[K] Kunita, H.: Stochastic integrals based on martingales taking values in Hilbert spaces, Nagoya Math. J. (1970).

[Ku] Kuo, H. H., White Noise Distribution Theory. Prob. and Stoch. Series, Boca Raton, FL: CRC Press, (1996).

[LP] Løkka, A., Proske, F.: Infinite dimensional analysis of pure jump Lévy processes on Poisson space. Preprint series, University of Oslo, No.15 (2002).

[LØP] Løkka, A., Øksendal, B., Proske, F.: Stochastic partial differential equations driven by Lévy space time white noise. To appear in Annals of Appl. Prob. (2003).

[MFA] Melnikova, I.V., Filinkov A.I., Anufrieva U.A.: Abstract Stochastic Equations 1, Classical and Distributional Solutions, Journal of Mathem. Sciences, Vol. 111, No. 2, (2002).

[MP] Meyer-Brandis, T., Proske, F.: On the existence and explicit representability of strong solutions of Levy noise driven SDE's with irregular coefficients, Preprint series, University of Oslo, No.15 (2004).

[MPe] Metiviér, M., Pellaumail, J.: Stochastic Intergration, Academic Press (1980).

[ØР] Øksendal, B., Proske, F.: White noise of Poisson random measures. To appear in Potential Analysis (2003).

[O] Obata, N.: White Noise Caculus and Fock Space. LNM 1577, Berlin, Springer-Verlag (1994).

[P] Proske, F.: The stochastic transport equation driven by space-time Lévy white noise. Preprint series No. 22, University of Oslo (2003).

[Sa] Sato, K.: Lévy Processes and Infinitely Divisible Distributions, Cambridge University Studies in Advanced Mathematics, Vol. 68, Cambridge University Press, Cambridge 1999.

[vG1] van Gaans, O.: A series approach to stochastic differential equations with infinite dimensional noise, Preprint, Leiden University.

[vG2] van Gaans, O.: Invariant measures for stochastic evolution equations with Hilbert space valued Lévy noise, Preprint, Leiden University.

[Z] Zhang, T.:Characterizations of white noise test functions and Hida distributions, Stochastics, Vol. 41 (1982), pp. 71-87. 
(Thilo Meyer-Brandis) Centre of Mathematics for Applications (CMA), Department of Mathematics, University of Oslo, P.O. Box 1053 Blindern, N-0316 Oslo, Norway.

E-mail address: meyerbr@math.uio.no 